\title{
ABDELMADJID ABOURA : UN ENSEIGNANT INTÉRESSANT ET UN CHERCHEUR IMPÉTRANT
}

\author{
Mustapha Guenaou \\ Centre de recherche en Anthropologie Sociale et Culturelle - CRASC, Orán
}

\begin{abstract}
Resumen
Esta nota es en memoria del profesor e investigador argelino Abdelmadjid Aboura, recientemente fallecido. Se resalta su contribución a la promoción de la lengua española en Argelia. Se repasa su trayectoria vital, empezando por la familia Aboura. Después su carrera académica en Tremecén, entre la enseñanza y la investigación científica. Finalmente, se incluyen sus obras más importantes.
\end{abstract}

Palabras clave: Abdelmadjid Aboura, Tremecén, ELE en Argelia.

\begin{abstract}
This note is in memory of the Algerian professor and researcher Abdelmadjid Aboura, who recently passed away. It highlights his contribution to the promotion of the Spanish language in Algeria. It reviews his life trajectory, starting with the Aboura family. Then his academic career in Tlemcen, between teaching and scientific research. Finally, his most important works are included.
\end{abstract}

Keywords: Abdelmadjid Aboura, Tlemcen, L2 Spanish in Algeria.

\section{Résumé}

Cette note est à la mémoire du professeur et chercheur algérien Abdelmadjid Aboura, récemment décédé. Elle souligne sa contribution à la promotion de la langue espagnole en Algérie. Elle passe en revue sa trajectoire de vie, à commencer par la famille Aboura. Puis sa carrière académique à Tlemcen, entre enseignement et recherche scientifique. Enfin, ses œuvres les plus importantes sont incluses.

Mots clés: Abdelmadjid Aboura, Tlemcen, l'espagnol comme L2 en Algérie.

A la suite du premier anniversaire de son décès, nous proposons cette contribution à la mémoire d'un enseignant intéressant et un chercheur impétrant : l'universitaire Abdelmadjid Aboura. Il avait contribué à la promotion de la langue espagnole, et ceci depuis ses débuts au lycée 
Docteur Benaouda Benzerdjeb ${ }^{1}$ de Tlemcen. Sa culture, ses connaissances en langues, son savoir et ses compétences pédagogiques ont été les principales motivations en faveur de la rédaction de cette contribution de l'histoire et la mémoire de la langue espagnole en Algérie en général et à Tlemcen en particulier.

\section{LA FAMILLE ABOURA}

Abdelmadjid Aboura est issu d'une vieille famille de Tlemcen, ancienne capitale du Maghreb central ${ }^{2}$. Connue pour son riche passé socio culturel $^{3}$, cette ville-médina était divisée en trois grands quartiers qui la composent : le quartier des hadars, le quartier des kouloughlis ${ }^{4}$ et le quartier des juifs. Les murailles ${ }^{5}$ faisaient de cette ville une cité medinale où les rues des métiers, des bijoutiers venaient accorder de l'importance et de la considération à une ville médiévale mais avec ses marqueurs de médina, une ville précoloniale pour les uns et une ville arabo andalouse ${ }^{6}$ pour les autres. Certains historiens parlent de ville musulmane dont la population est majoritairement de religion musulmane ${ }^{7}$. A cette ville est liée un grand centre commercial, communément appelé El Qissariya ${ }^{8}$. Elle est le creuset de la culture arabo musulmane'.

Appelé hawmat el hdar (quartier des hadars), le quartier est constitué de familles autochtones, d'origine arabo andalouse dont leur origine remonte à la date de I492, qui coïncide avec la chute de Grenade et l'exil massif des Andalous. D'ailleurs, elles sont arrivées avec leurs traditions

1 Docteur Benaouda Benzerdjeb (1921-1956), un médecin généraliste formé en France (Montpellier et Paris), tombé au champ d'honneur, le 17 janvier 1956 au douar ouled Hlima, près de Sebdou (Tlemcen).

2 Dhina, Atallah, Les états de l'occident musulman aux XIII', XIV' et XV siècles. Alger, OPU-ENAL, 1984. Idem, Le royaume Abdelouadide à l'époque d'Abou Hammou Moussa 1er et d'Abou Tachfin 1er, Alger, OPU-ENAL, 1985.

3 Benkelfat Djelloul, Il était une fois TLEMCEN... récit d'une vie, récit d'une ville, Tlemcen, Editions Ibn Khaldoun, 2002.

4 Andosian Sossie, «Tlemcen, un espace de métissage », dans Expériences du Divin dans l'Algérie contemporaine; adeptes des saints dans la région de Tlemcen, Paris, CNRS, 2001, pp. 39-53.

5 Bouali, Sid Ahmed, Les deux grands sièges de Tlemcen, Alger, ENAL, 1984.

6 Dhina Atallah, op. cit.

7 Bel Alfred, Population musulmane de Tlemcen, Paris, Lib. Paul Geuthner, 1908.

8 Andosian, op.cit.

9 Addou Ghizlane, Tlemcen chants et adages, Alger, Manal, 1994. 
et leurs métiers, ceux qui étaient la fierté de Tlemcen ${ }^{10}$. Ce quartier est situé au bas de la Qissariya ${ }^{11}$.

Le deuxième quartier est celui des juifs. Il est appelé Derb El Yhoud, un petit quartier où vivaient les juifs, devenus des citoyens français, avec le décret Cremieux ${ }^{12}$. Ils étaient attachés à leur culture, leur culte et leurs activités économiques dont certains petits métiers dans des ruelles respectives de la médina. Ils étaient à Tlemcen ${ }^{13}$, depuis l'installation en ville du Rabb Ankawa ${ }^{14}$, alors enterré à la sortie de Tlemcen, près de la porte dite Beb El Qarmadine ${ }^{15}$. Sa tombe est un lieu de visites pieuses des touristes étrangers d'origine juive.

Le troisième quartier est celui des Kououghlis. Il était connu sous l'appellation de hawmat El Qraghla, dans le sens de quartier des kouloughlis. Leur arrivée à Tlemcen remonte au Xvi ${ }^{\circ}$ siècle, à l'arrivée des Ottomans en Algérie. Installés au niveau du territoire national, Ils étaient des militaires de la Régence d'Alger. Engagés dans l'armée de l'Empire ottoman, sous le commandement des frères Barberousse ${ }^{16}$. Engagés comme soldats des Janissaires, ils ne pouvaient pas se marier ou être mariés. Ils respectaient les clauses du contrat d'engagement.

A la fin de leur contrat, ces anciens militaires étaient rendus libres. Ils pouvaient crée des activités, qu'elles soient artisanales, commerciales ou autres. Ils ne pouvaient occuper des postes administratifs, après leur fin

10 Barges, J-J-L, Complément de l'histoire des Beni-Zeiyan, rois de Tlemcen. Alger, Dar El Alif, 2011 ; Idem, Tlemcen, ancienne capitale du royaume de ce nom. Souvenirs d'un voyage, Alger, Dar E1 Alif, 2011 ; Idem, Histoire des Beni Zeiyan. Rois de Tlemcen. Par l'imam Sidi Abou Abdallah Mohammed Ibn Abdaljalil Ettenissy, Paris, Benjamin Duprat Lib., 1852.

11 Andosian, op.cit.

12 Du nom d'Adolphe Crémieux (1796 -1880), ce décret est promulgué pour attribuer la citoyenneté française aux juifs d'Algérie.

13 Lachachi Hadj Omar, Le passé prestigieux de Tlemicen. Ancienne capitale du célèbre berbère Ya'Ghmorac'en, fondateur de la nation. Tlemcen, Editions Ibn Khaldoun, 2002.

14 Ephraim Al Naqawa (1359-1442), rabbin, et médecin qui serait un théologien séfarade. Il est enterré dans le cimetière, communément q-bour El yhoud à Qbaça, près de la porte de Beb El Qarmadine.

15 Cette porte conduit au hawz de Tlemcen : Benabadji Fodil. Tlemcen dans l'histoire à travers les contes et légendes, Paris, Publisud, 2003.

16 Il s'agit d'une fratrie de corsaires :

- Baba Aroudj (vers 1774 -1518 entre Tlemcen et Oran).

- Kheir Eddine (vers 1466 -1546)

- Ishaq ( ?- ?) 
d'engagement militaire. Ils firent plusieurs métiers et plusieurs professions. Puis, ils pouvaient se marier avec les femmes du pays.

Ce mariage mixte avait donné le qualificatif de kouloughli dans le sens attribué à la descendance, issue d'un mariage mixte entre un Ottoman et une Algérienne. Dans le cadre d'une enquête sur les patronymes ${ }^{17}$, nous avons relevé une liste de familles d'origine kouloughlie ${ }^{18}$. La famille Aboura en fait partie. D'ailleurs, sa famille est connue sous l'appellation locale «Dar Ben 'Aboura $»^{19}$.

Sa famille est connue, au niveau local, pour son rang social puisque nombreux sont ses membres qui font partie de l'élite locale, celle des instruits et des lettrés. A titre illustratif, nous évoquons les deux intellectuels de la famille du XX $\mathrm{XX}^{\circ}$ siècle : Mostefa Aboura $^{20}$ et son fils Kheir Eddine ${ }^{21}$. Le père est l'un des premiers instituteurs algériens musulmans, formés à l'Ecole des Instituteurs ou Ecole Normale de Bouzeriah (Alger); et le fils, un ancien employé de la radio locale, ayant effectué de la recherche au service de l'Etat civil de la ville de Tlemcen.

Ce dernier, l'histoire et la mémoire lui reconnaissent le travail effectué dans les archives de la mairie de Tlemcen pour mettre à la disposition des chercheurs musicologues et historiens, avertis et profanes, une liste des musiciens et interprètes de la musique arabo andalouse. Sur cette liste figuraient les musulmans et les juifs, versés dans cet art.

\section{Parcours D'abdelmadjid aboura}

En 1952 à Tlemcen ${ }^{22}$, Abdelmadjid est venu égayer le foyer de «Dar

17 Nous avons utilisé deux anciens dictionnaires : Youssouf, R, Dictionnaire Turc-français (en caractère latin), Constantinople, Imprimerie Impériale, 1888 ; Barbier De Meynard, Charles, Dictionnaire Turc-français (en caractère latin), Paris, Ernest Leroux, 1881.

18 Charif Ghouti, L'arbre de Tlemcen. Tlemcen, Imprimerie Sari, 1993.

19 Dar Ben 'Aboura signifie la famille Aboura. Dans le parler tlemcenien, 'aboura signifie jeune brebis.

20 Le père et le fils Aboura :

- Mostefa Aboura (1875-1935), ancien élève de l'école normale de Bouzeriah, devenu instituteur à l'école Descieux de Tlemcen.

- Kheir Eddine Aboura (1908-1977), ancien directeur artistique à la station de la radio de Tlemcen puis adepte de l'ordre religieux à la zaouia de Benaouda Benmamcha.

21 El Hassar Benali, Tlemcen dans les textes, Alger, ENAG, 2011. Idem et al., Tlemcen Florilège. Histoire, Art, Politique, Portraits et scènes de vie, Alger, Dalimen, 2011.

22 Sari Djilali, A la recherche de notre histoire, Alger, La Casbah, 2003 ; Idem, L'émergence de 
Ben 'Aboura ». Ce patronyme est attribué à cette famille, à la suite de la promulgation de la loi de l'état civil de 1882 . Une commission a été constituée pour attribuer des patronymes aux familles de la ville de Tlemcen et ses environs ${ }^{23}$ (le hawz ${ }^{24}$ : Ain E1 Houts, Ouzidan, E1 Eubbad, etc.).

Comme tous les enfants de sa génération, le jeune Abdelmadjid a été inscrit à l'école primaire pour son instruction de base. Son inscription remonte à la période de la guerre de la libération nationale. L'instruction était devenue obligatoire pour les garçons. Très peu de fillettes étaient inscrites pour poursuivre une scolarité solide et approfondie.

Dans les années soixante, il a été reçu à l'examen de la sixième. Cet examen lui a permis de poursuivre ses études dans le collège. A cette époque, il y avait peu d'établissements scolaires : deux lycées et deux collèges. Les deux lycées et les deux collèges refusaient la mixité. Pour les deux lycées, nous parlons du lycée de jeunes filles qui, baptisée à l'indépendance nationale, devient Lycée Maliha Hamidou. Et, l'autre, le Grand Lycée de Tlemcen, devenue après I962, lycée de garçons Docteur Benaouda Benzerdjeb.

C'est dans ce lycée que j'avais connu Abdelmadjid Aboura, élève en classe littéraire, dans la branche Espagnol. Il excellait dans cette langue hispanique, depuis son jeune âge, comme ce fut le cas de Nordine Malki ${ }^{25}$, un autre hispanisant. Je l'avais rencontré pour la première fois, à la grande cour du lycée, en présence de deux enseignants dont le premier était son professeur d'espagnol, en l'occurrence M. García, un républicain refugié à Oran, en 1939. L'autre, un enseignant hispanisant qui nous enseignait la langue française : L'abbé Alfred Berenguer ${ }^{26}$, ancien curé à Montagnac, actuellement Remchi dans la wilaya de Tlemcen.

Ayant obtenu son baccalauréat, Abdelmadjid aurait été inscrit à l'université d'Oran pour poursuivre ses études supérieures en langue. Titulaire

l'intelligentsia algérienne (1850-1950), Alger, ANEP, 2006 ; Idem, Tlemcen et ses élites, Alger, Editions Casbah, 2011 ; Tlemcen la zyanide, Alger, Editions Casbah, 2011.

23 Collectif, Tlemcen et sa région, Paris, Richesses de France, 1954.

24 Andosian, Sossie, op.cit.

25 Un autre hispanisant, Un enseignant chercheur à l'université d'Oran Essenia, devenu directeur du Musée National Ahmed Zabana d'Oran. Il a été responsable dans plusieurs titres de la presse locale. Une biographie est en chantier.

26 Un enseignant d'origine espagnole et hispanisant qui mérite une notice pour l'histoire et la mémoire de la langue espagnole en Algérie. 
d'une licence, il devient enseignant de la langue espagnole dans sa ville natale. Il aurait poursuivi ses études en langue avec beaucoup de succès.

Attaché à la recherche scientifique, Abdelmadjid Aboura assistait aux différents événements, organisés par le Centre de Recherche en Anthropologie Sociale et Culturelle à Oran. D'ailleurs, il pensait que CRASC est un centre du savoir scientifique que les chercheurs sont appelés à le fréquenter pour l'enrichissement de leur connaissances scientifiques et culturelles.

ECOLYMET est une association des anciens élèves de la ville de Tlemcen dont les anciens élèves du Lycée Docteur Benzerdjeb. Elle organise, annuellement, au mois de mai une journée de retrouvailles des anciens élèves des établissements scolaires de Tlemcen ${ }^{27}$. Il avait assisté à plusieurs éditions au niveau local.

Centre des Etudes Andalouses, alors créé à Tlemcen après la grande manifestation de «Tlemcen, capitale de la culture islamique » (2OII), avait organisé des rencontres qu'Abdelmadjid Aboura appréciait.

Il a, par ailleurs, assisté à plusieurs reprises aux manifestations, organisées au niveau du Palais de la Culture de Tlemcen et à la Maison de la culture Abdelkader Alloula de la même ville. Il avait assisté aux premières journées d'Ain El Houts, manifestation ayant connu peu d'éditions pour des raisons logistiques. D'ailleurs, à cette première édition, avait assisté le chef spirituel de la tariqa El Allawiya de Mostagamen, en compagnie de plusieurs adeptes de la zaouia. Il s'agit de Cheikh Khaled Bentounes ${ }^{28}$.

Au début des années soixante-dix, la ville de Tlemcen était dotée de trois bibliothèques publiques où les élèves et les étudiants originaires de la ville venaient se ressourcer : Bibliothèque pédagogique, le centre culturel français et le centre culturel du parti. Abdelmadjid fréquentait ces trois lieux de lecture et de culture. Ils constituaient un triangle de la culture et de la lecture des élèves de Tlemcen.

27 Ecolimet, Journée commémorative en l'honneur des collégiens, Médersiens, Lycéens et Etudiants de Tlemcen, Tlemcen, [s.n.], 2001 ; Idem, Retrouvailles des élèves de l'EPS, du Collège de Slane, du Lycée de garçons et de la Médersa, Tlemcen, [s.n.], 2003 ; Idem, Centenaire de la medersa de Tlemcen 1905-2005. Tlemcen, [s.n.], 2005.

28 Sheikh Khaled Bentounes (né 1949), devenu depuis 1975 le guide spirituel de la tariqa d'E1 alawiya. Il est auteur et co auteur de plusieurs publications. 
Un petit groupe de lycéennes et de lycéens était constitué au sein du centre culturel du parti. Grâce la bonne volonté du directeur du centre, en l'occurrence M. Ait Salem, une commission culturelle activait sous l'égide du commissariat politique du FLN à Tlemcen. Cette commission avait donné une opportunité aux jeunes, en les autorisant à organiser des petites rencontres : tous les samedis, une conférence était donnée par les élèves des deux lycées à Tlemcen. C'était les prémices d'une mixité non publiques $^{29}$ Abdelmadjid venait pour assister à ces rencontres - débats.

\section{Abdelmadjid Aboura, entre l'enseignement et la recherche SCIENTIFIQUE}

En 1992, il a été un enseignant vacataire à l'Institut de la culture populaire, qui dépendait de l'Université de Tlemcen, avant de devenir Université Abou Bekr Belkaid de Tlemcen. Il enseignait le module d'espagnol aux étudiants du Magister de l'Institut de la Culture Populaire. Puis, il se présenta au concours du Magistère. Réussi au concours, il prépara son Magister qu'il avait soutenu pour poursuivre ses études en doctorat. Recruté à l'Université Abou Bekr Belkaid, il devient enseignant - chercheur.

Nombreux sont les étudiants qui lui reconnaissent ses compétences en langue espagnole. Il a été d'un grand apport dans la contribution et l'amélioration de la formation et de l'apprentissage en langue. Les jeunes hispanistes et hispanisants de l'Université Abou Bekr Belkaid de Tlemcen, en gardent de bons souvenirs. Il aurait une part dans la contribution dans les échanges universitaires : l'exemple des accords signés avec l'université de Murcie (Espagne).

29 Les jeunes, filles et garçons, animaient et assistaient à ces rencontres hebdomadaires dans une salle de lecture de la bibliothèque du Parti, sur la grande place de la ville de Tlemcen. Nombreuses étaient les filles qui assistaient à ces rencontres. A cet effet, je me permets d'évoquer les noms des premières jeunes filles qui assistaient avec nous : Certaines étaient des élèves du Collège De Salane, devenu CEM ibn Khaldoun. Je cite :

- Rabia Bekkar, aujourd'hui une grande sociologue e=dans une université française

- Fatiha S.

- Bahia A.

- Farida S.

- Rabea K.

- Houria

- Etc. 
Dans le cadre de sa mission et ses fonctions, en relation avec l'encadrement des étudiants, il a été directeur et co-directeur de thèse. Il avait, également, aidé ses étudiants à faire valoriser leurs recherches scientifiques en les faisant publier dans la revue du laboratoire Anthropologie des religions comparées de l'Université Abou Bekr Belkaid de Tlemcen.

En sa qualité d'enseignant-chercheur, il fit des recherches dans un domaine précis à savoir le soufisme, communément appelé «Ettassawouf ». Ce domaine porte sur la voie mystique, la vision ésotérique : la voie mystique en Islam.

Il se spécialisa dans une branche du soufisme : El fardanya. Il s'est beaucoup investi jusqu'à créer un site consacré à sa recherche sur cette voie mystique, très peu connue par les chercheurs, les spécialistes du soufisme ou mystique en Islam. Selon ses propos, lors de nos différentes rencontres, la fardaniya est la voie la plus importante dans le soufisme musulman. Cette voie serait fondée par Sheikh Kaddour Benachour ${ }^{30}$ qui, en chef mystique et poète soufi, avait transmis les secrets de cette voie à Sidi Benaouda Benmamcha ${ }^{31}$, chef de zaouïa, située à Derb Sidi $\mathrm{Amrane}^{32}$, dans la hawmat El Qaraghila (Tlemcen). La zaouïa est l'espace de méditation et le lieu des séances des auditions spirituelles ${ }^{33}$.

Il prépara son mémoire de Magister qu'il avait soutenu, en 2002, devant un jury à l'université d'Oran Es Senia. Cette étude lui ouvrit le chemin de la recherche scientifique dans le domaine du soufisme islamique. Il était Maitre de conférences, Habilité à diriger la recherche. Dans sa spécialité au niveau du département, il est chercheur dans le

30 Sheikh Kaddour Benachour (1850-1938) ; sa zaouïa se trouvait à Nedroma, lieu de sa sépulture. Il a beaucoup d'adeptes et de fidèles dont un grand nombre se recueille sur sa tombe. Ces derniers avait adopté et suivi la voie, la tariqa qu'il avait tracé par ;

- Son savoir mystique

- Sa conviction

- Son engagement dans l'enseignement des principes de la Fardaniya.

Son enseignement mystique vise la recherche, par le biais de la voie mystique, la Grande Vérité d'Allah. Il avait instauré des " madjma' », dans le sens d'un rituel de séances d'audition spirituelle. D'ailleurs, un qualificatif lui est attribué ; Sheikh Kaddour Benachour El Ferdany.

31 Chef de la zaouïa, située à Derb Sidi Amran, dans le quartier des Kouloughlis, Sheikh Benaouda Benmamcha (1888-1983).

32 Témoignage de F. Benahmed, aujourd'hui décédée. Il n'habitait pas loin du siège de la zaouïa. 33 Andosian, op.cit. 
domaine de la didactique : didactique du français, sémiotique des textes, littérature comparée et analyse du discours.

Titulaire d'un doctorat, il a été maitre de conférences au département de l'espagnol au niveau de la faculté des Lettres de l'Université Abou Bekr Belkaîd de Tlemcen. Il avait encadré plusieurs travaux d'étudiants. Il était, également, directeur de recherche à l'EDAF ${ }^{34}$. Il figure sur l'annuaire de $\mathrm{LaFEF}^{35}$.

Devenu enseignant-chercheur, il s'est versé dans la recherche scientifique pour faire valoir les résultats de ses investigations dans le domaine de la voie mystique de la Fardaniya. Il était sollicité pour donner des conférences, animer des séances radiophoniques, et surtout à participer à des rencontres scientifiques, des séminaires et des colloques.

Il serait l'initiateur du projet du colloque international, lors de la grande manifestation « Tlemcen, capitale de la culture islamique », tenu à Tlemcen durant l'année 2oIr. Intitulé «soufisme et altérité chez le saint gnostique Sidi Kaddour Benachour El Ferdany ", ce colloque, tenu du I3 au I5 juin 20II, a été pris en charge par l'équipe ETHOMED du Laboratoire Anthropologie des religions comparées de l'université Abou Bekr Belkaid de Tlemcen.

Convaincu, Abdelmadjid Aboura créa un site pour faire valoir les résultats de ses recherches et ses grandes investigations dans le domaine du «tsassawuf ». Il s'intéressa à Sidi Boumediene Shoaib dont le mausolée domine la ville de Tlemcen, sur les hauteurs d'El Eubbad, non loin de la mosquée porte son nom. Il menait ses recherches comme son contemporain Hikmet Sari, un docteur en médecine versé dans le soufisme et la recherche sur l'histoire et les fondements de la voie du saint patron de la ville de Tlemcen, Sidi Boumediene ${ }^{36}$.

Il était un habitué du Café maure "Quahwet El Bouri », lieu préféré de ses rencontres avec des amis et des chercheurs, avertis et profanes. Comme tous les Tlemceniens, il aimait retrouver ses amis, après la prière d'El 'Asr. D'ailleurs, le café maure, qu'il fréquentait, nétait pas loin de la mosquée.

34 Ecole Doctorale Algéro Française.

35 Langue Française et Expressions Francophones.

36 Sekkal Sid-Ahmed, Un saint de première grandeur :Sidi Abou-Madian de Tlemcen, Tlemcen, Imprimerie Sekkal, 1993. 
Le décès D'Abdelmadjid Aboura

Il était fumeur ; il eut des soucis cardiaques. Abdelmadjid Aboura est décédé le 9 du mois de mai $2019^{37}$ à Tlemcen. L'université algérienne avait perdu un enseignant de valeurs. Il avait laissé, en effet, un grande vide dans le domaine de la recherche scientifique sur la didactique et les connaissances relatives aux voies ou tariqa-s du soufisme musulman en Algérie et surtout l'approfondissement des connaissances sur la « Ferdaniya » ( l'Unicité), mystique basée sur les fondement de la recherche de la Grande Vérité d'Allah.

CEuvres d’Abdelmadjid Aboura

a- Thèse

- «Aspects et fonctions du récit initiatique dans la tradition théosophique de l'Islam » (Magister, 2002)

- Etudes des processus didactiques et cognitifs dans l'acquisition de la littéracie et des compétences interculturelles. Constitution d'un modèle de situation de l'acquisition des compétences inter- discussives et interculturelles chez les apprenants plurilingues maghrébins (Doctorat, 2012).

\section{b- Conférences}

- «Soufisme et altérité chez le saint gnostique Sidi Kaddour Benachour El Ferdany », un colloque international, tenu du I3 au I5 juin 20II, organisé par l'équipe ETHOMED du Laboratoire Anthropologie des religions comparées de l'université Abou Bekr Belkaid de Tlemcen.

- «Les érudits de Bejaia au Moyen- âge », organisé le mois de mai 2016 par l'association culturelle et éco touristique des Aiguades à Bejaia. Il anima une conférence intitulé « La gnose poétique de Sidi Abû Medyan Shu'ayb».

37 Hadef Sarra Ikram, "Nécrologie ; Aboura Abdelmadjid" dans Revista Argelina, 2019, num. 9, pp 97-98. 
c- Animations radiophoniques

- Entretien accordé à la Radio Algérie Internationale, en avril 2018

d-Articles scientifiques

- L'oralité initiatique dans le soufisme littéraire.

- Etude onomastique et territorialisation de l'espace toponymique : le cas de la ville de Tlemcen

- Aspects et fonctions du récit initiatique dans la tradition théosophique musulmane : le personnage initiatique en genèse. bonne.

- Concept de Wihdat el Wujud chez In Tophail et Moise de Nare- Publication d'ouvrages

- Le vivant fils de l'Eveillé ou la grande triade soufie (Allemagne), EUE Omniscription, 20II.

- Le régime du solitaire dans le soufisme unitif étude comparative : aspects et fonction du récit initiatique dans la littérature soufie (Allemagne), EUE Omniscription, 2oro. 
